\title{
Detection of patients with chronic thromboembolic pulmonary hypertension by volumetric iodine quantification in the lung-a case control study
}

\author{
Jan Robert Kroeger ${ }^{1,2} \wedge$, Jakob Zöllner ${ }^{2}$, Felix Gerhardt ${ }^{3}$, Stephan Rosenkranz ${ }^{3}$, Roman Johannes Gertz ${ }^{2}$, \\ Shir Kerszenblat ${ }^{4}$, Gregor Pahn ${ }^{5}$, David Maintz ${ }^{2}$, Alexander C. Bunck ${ }^{2}$ \\ ${ }^{1}$ Department of Radiology, Neuroradiology and Nuclear Medicine, Johannes Wesling University Hospital, Ruhr University Bochum, Germany; \\ ${ }^{2}$ Department of Radiology, Faculty of Medicine and University Hospital Cologne, University of Cologne, Cologne, Germany; ${ }^{3}$ Department of \\ Cardiology, Faculty of Medicine and University Hospital Cologne, University of Cologne, Cologne, Germany; ${ }^{4}$ Philips Healthcare, Haifa, Israel; \\ ${ }^{5}$ Philips Healthcare, Hamburg, Germany
}

Contributions: (I) Conception and design: JR Kroeger, S Rosenkranz, D Maintz, AC Bunck; (II) Administrative support: S Rosenkranz, D Maintz, S Rosenkranz; (III) Provision of study materials or patients: F Gerhardt, S Rosenkranz, S Kerszenblat, G Pahn; (IV) Collection and assembly of data: JR Kroeger, J Zöllner; (V) Data analysis and interpretation: JR Kroeger, J Zöllner, AC Bunck, RJ Gertz; (VI) Manuscript writing: All authors; (VII) Final approval of manuscript: All authors.

Correspondence to: Jan Robert Kroeger, MD. Department of Radiology, Neuroradiology and Nuclear Medicine, Johannes Wesling University Hospital, Ruhr University Bochum, Hans-Nolte-Str.1, 32429 Minden, Bochum, Germany. Email: janrobert.kroeger@muehlenkreiskliniken.de.

Background: To evaluate whether volumetric iodine quantification of the lung allows for the automatic identification of patients with chronic thromboembolic pulmonary hypertension (CTEPH) and whether the extent of pulmonary malperfusion correlates with invasive hemodynamic parameters.

Methods: Retrospective data base search identified 30 consecutive patients with CTEPH who underwent CT pulmonary angiography (CTPA) on a spectral-detector CT scanner. Thirty consecutive patients who underwent an identical CT examination for evaluation of suspected acute pulmonary embolism and had no signs of pulmonary embolism or PH, served as control cohort. Lungs were automatically segmented for all patients and normal and malperfused volumes were segmented based on iodine density thresholds. Results were compared between groups. For correlation analysis between the extent of malperfused volume and mean pulmonary artery pressure (mPAP) and pulmonary vascular resistance (PVR) 3 patients were excluded because of a time span of more than 30 days between CTPA and right heart catheterization.

Results: Patients with CTEPH had a higher percentage of malperfused lung compared to controls $(43.25 \% \pm 24.72 \%$ vs. $21.82 \% \pm 20.72 \% ; \mathrm{P}=0.001)$ and showed reduced mean iodine density in malperfused and normal-perfused lung areas, as well as in the vessel volume. Controls showed a left-tailed distribution of iodine density in malperfused lung areas while patients with CTEPH had a more symmetrical distribution (Skew: $-0.382 \pm 0.435$ vs. $-0.010 \pm 0.396 ; \mathrm{P}=0.004$ ). Patients with CTEPH showed a significant correlation between the percentage of malperfused lung volume and the PVR ( $r=0.57, \mathrm{P}=0.001)$.

Conclusions: Volumetric iodine quantification helps to identify patients with CTEPH by showing increased areas of malperfusion. The extent of malperfusion might provide a measurement for disease severity in patients with CTEPH.

Keywords: Hypertension; pulmonary; computed tomography angiography; chronic thromboembolic pulmonary hypertension (CTEPH); dual-energy CT (DECT)

\footnotetext{
$\wedge$ ORCID: 0000-0003-1218-7610.
} 
Submitted Apr 24, 2021. Accepted for publication Aug 23, 2021.

doi: 10.21037/qims-21-229

View this article at: https://dx.doi.org/10.21037/qims-21-229

\section{Introduction}

Pulmonary hypertension $(\mathrm{PH})$ describes a group of diseases that are defined by an elevated pressure in the pulmonary vasculature. The WHO classification subdivides $\mathrm{PH}$ in different groups based on pathogenesis and classifies chronic thromboembolic pulmonary hypertension (CTEPH) as WHO group 4 (1). The pathogenesis of CTEPH is based on acute pulmonary emboli that persist either because they are not detected or do not dissolve under anticoagulant therapy and endothelialized residues remain leading to significant stenoses or complete obstruction of pulmonary vessels, a condition called chronic thromboembolic disease (CTED). If these residues lead to a pathologic increase in pulmonary artery pressure the condition is called CTEPH (2). CTEPH is associated with a significant mortality with a median survival rate of $10-20 \%$ at $2-3$ years if not treated (3). The identification of patients with CTEPH is important because they can potentially be cured by surgical thrombendarteriectomy. Moreover balloon angioplasty can be used to improve vessel patency in selected patients (4). The diagnosis of $\mathrm{PH}$ is made by right heart catherization (RHC) and a mean pulmonary artery pressure (mPAP) of above $20 \mathrm{mmHg}$ in combination with a pulmonary vascular resistance (PVR) of at least 3 Wood Units is considered as definitive $\mathrm{PH}(5,6)$. RHC can also discern pre-capillary and post-capillary $\mathrm{PH}$ by evaluating the pulmonary artery wedge pressure (PAWP) (5). CTEPH is a form of precapillary $\mathrm{PH}$ with a PAWP of less than $15 \mathrm{mmHg}$. For further differentiation of the different groups of precapillary $\mathrm{PH}$ (WHO Group 1, 3-5) further diagnostic tests including V/Q scintigraphy, lung function testing, CT pulmonary angiography (CTPA), conventional angiography are used (6).

The severity of $\mathrm{PH}$ is defined by clinical performance and hemodynamic parameters measured by RHC (5).

While CTPA is the standard of care for diagnosis of acute pulmonary embolism (7), V/Q-scintigraphy has long been favored in the evaluation of $\mathrm{PH}$ because of its ability to provide information on pulmonary perfusion instead of merely showing vessel anatomy $(8,9)$. The diagnosis of CTEPH by CTPA can be challenging because the vessel abnormalities may be minor and thus even experts reach only a suboptimal sensitivity (10). The introduction of dual-energy CT (DECT) has enabled the reconstruction of iodine density images (IDIs) which provide information about iodine distribution and concentration. Therefore, a comprehensive analysis of pulmonary perfusion and vessel anatomy using standard clinical CT protocols is possible without the need for additional scans (11-14). IDIs can show different types of perfusion abnormalities that have a different prevalence in different types of $\mathrm{PH}(15-17)$. This is especially helpful in the diagnosis of CTEPH with a reported sensitivity of up to $100 \%$ (17).

A software application, that has recently become available, now allows for volumetric iodine quantification per voxel for a $3 \mathrm{D}$-dataset acquired on a dual-layer CT platform called spectral-detector CT (SDCT). The objective of our study is to evaluate whether these volumetric iodine quantifications can help diagnose CTEPH. Furthermore, we evaluated if there is a correlation between hemodynamic parameters and the extent of pulmonary malperfusion on IDIs.

We present the following article in accordance with the STROBE reporting checklist (available at https://dx.doi. org/10.21037/qims-21-229).

\section{Methods}

\section{Patient population}

This retrospective study was approved by the Ethics Commission of Cologne University's Faculty of Medicine and the need for informed consent for study enrollment was waived due to the retrospective nature of this study. The study was conducted in accordance with the Declaration of Helsinki (as revised in 2013).

A retrospective data base search identified 98 consecutive patients that underwent CTPA for the evaluation of possible $\mathrm{PH}$ on a spectral detector CT (SDCT) between June 2016 and February 2018. All these patients had suspected $\mathrm{PH}$ and were admitted to the department of cardiology at our hospital for further testing. Additional testing in accordance with the 2015 ESC/ERS guidelines for the diagnosis of $\mathrm{PH}$ was performed (5). Patients were classified by expert consensus based on all available diagnostic tests and CTEPH was diagnosed in 30 of these patients. In 3 of these patients there was a delay of more than 30 days between CT examination and RHC, thus we excluded these 
patients from correlation analysis between CT and RHC parameters. For the other 27 patients the median time between CT examination and RHC was 1 day (Min: 0 days, Max: 23 days).

In addition to that a cohort of patients that underwent CTPA on the same CT scanner in the same time span for evaluation of suspected acute pulmonary embolism was identified. Of these patients, 30 consecutive patients in which pulmonary embolism was ruled out and who did not show signs of $\mathrm{PH}$ or extensive parenchymal infiltration were selected as control cohort.

\section{Image acquisition and reconstruction}

CT data were acquired on a 128-row spectral detector CT (IQon, Philips Healthcare, Best, The Netherlands). According to our institutional standard for CTPA studies, all patients received $50 \mathrm{~mL}$ of contrast media (300 mg Iodine/ $\mathrm{mL}$, Accupaque, GE Healthcare, Chicago, Illinois, USA) followed by a $40 \mathrm{~mL} \mathrm{NaCl}$ flush injected intravenously with a flow rate of $4 \mathrm{~mL} / \mathrm{s}$. Scanning was initiated with a delay of $4.9 \mathrm{~s}$ after an attenuation of $150 \mathrm{HU}$ was reached in the MPA. Scanning and reconstruction parameters were: slice collimation $0.625 \mathrm{~mm}$; rotation time $0.33 \mathrm{~s}$; tube potential $120 \mathrm{kV}$, automatic tube current modulation; reconstructed axial slice thickness $1 \mathrm{~mm}$; reconstructed axial slice overlap $0.5 \mathrm{~mm}$; pixel matrix $512 \times 512$. Spectral information in a so called spectral-based-image (SBI) format was saved.

\section{Image analysis}

Automatic segmentation of the whole lung (including lung parenchyma, smaller airway and pulmonary vasculature) was performed using commercially available software (IntelliSpace Portal, COPD tool, Philips, Best, The Netherlands). Automatic segmentation was controlled and edited when needed. Segmentation and SBI file were exported to a dedicated software for volumetric iodine quantification (Volumetric quantification, Philips Intellispace Release 11). Lung volume was then separated into 3 different compartments based on iodine density (measured in $\mathrm{mg} / \mathrm{mL}$ ). For this, different settings were tested and correlated to the visual assessment by two radiologists with [6 and 12 years of experience in $\mathrm{PH}$ imaging (blinded)]. Finally, the vessel compartment was defined as all voxels with an iodine density greater than half of the iodine density measured in a ROI in the left atrium. This was done to account for pulmonary arteries and veins. Malperfused lung volume was defined as voxels with an iodine density of less than $5 \%$ of the iodine density in the main pulmonary artery. An example of the lung volume separation is presented in Figure 1. Volume, volume percentage and mean iodine density, as well as skewness of iodine density were recorded for all patients. Skewness describes the asymmetry of the iodine density distribution, with more negative values indicating a shift of the peak number of voxels towards higher iodine density values and more positive values indicating a shift of the peak number of voxels towards lower iodine density values. A skewness of zero is consistent with a symmetric distribution of iodine density values around the mean in a given volume.

\section{Statistics}

Statistical analysis was performed using SPSS (Version 25, IBM). Values are given as mean \pm standard deviation. Visual analysis of histograms of data samples showed that our data was not normally distributed. $95 \%$ CI were calculated using bias corrected and accelerated bootstrapping with 1,000 iterations to account for the non-normal distribution. To compare differences between groups non-parametric testing was used. Significance of differences was calculated by Mann-Whitney-U-test. A P value of less than 0.05 was accepted as statistically significant. Cohen's d was calculated to illustrate effect sizes. Correlation between the extent of malperfusion and hemodynamic parameters was calculated using Spearman's-rho-correlation coefficient. ROC analysis was performed for the prediction of existing CTEPH with the percentage of malperfused lung volume. Youden's index was used to calculate a cut-off value and define sensitivity and specificity at the optimal cut-off point.

\section{Results}

Patients with CTEPH had a mean age of $59.17 \pm 17.02$ (95\% CI: 52.81-65.52) while patients in the control group had a mean age of $59.80 \pm 18.23$ (95\% CI: 52.99-66.61) and the difference was not significant $(\mathrm{P}=0.865)$.

MPAP was elevated in all patients with CTEPH with $43.32 \pm 11.30 \mathrm{mmHg}$. PVR in patients with CTEPH was $8.12 \pm 3.78$ Wood units.

Patients with CTEPH had a significantly higher percentage of malperfused lung volume compared to controls. Although CTEPH may be associated with pulmonary artery stenosis and obliteration, the percentage of vessel volume was not significantly different between 

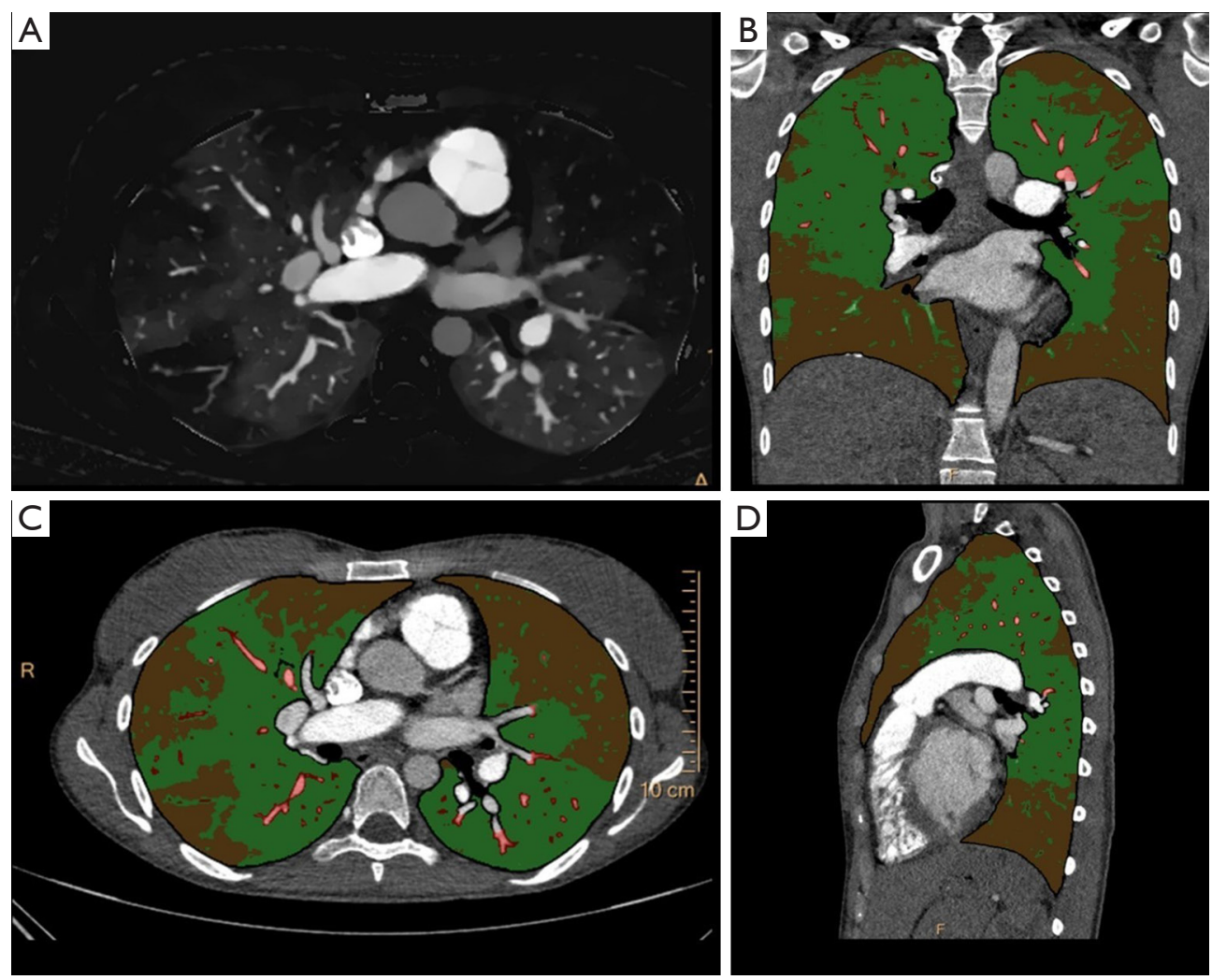

Figure 1 Exemplary assessment of iodine density-based lung segmentation in a patient with chronic thromboembolic pulmonary hypertension. (A) Iodine density image. (B-D) Color-coded images from the automatic quantification based on iodine density. Color coding as follows: red, vessel; green, normal perfused lung regions; brown, malperfused lung regions.

patients with CTEPH and controls (see Figure 2). Patients with CTEPH showed a significantly lower iodine density in malperfused and normal-perfused lung areas as well as in the vessel volume. However, the differences between patients with CTEPH and controls for iodine density values were relatively small and showed only moderate effect size (Cohen's d). In the control group the iodine density in malperfused lung areas showed a negative skew, owing to a left skewed distribution, which means that voxels tended to have a higher iodine density. In patients with CTEPH the distribution of iodine density in malperfused lung areas was more uniform. The difference in skew between patients with CTEPH and controls was statistically significant (see Figure 3). The results for comparison of patients with CTEPH and controls are summarized in Table 1.

Patients with CTEPH showed a significant correlation between PVR and the percentage of malperfused lung volume $(\rho=0.58, \mathrm{P}=0.001)$ while the correlation between $\mathrm{mPAP}$ and the percentage of malperfused lung volume was not significant $(\rho=0.34, P=0.079)$ (see Figures 4,5).
ROC analysis for the prediction of CTEPH by using the percentage of malperfused lung volume produced an AUC value of 0.76 (see Figure 6). By using Youden's index a cutoff point of $\geq 36.45 \%$ was identified resulting in a sensitivity of 0.67 and a specificity of 0.8 .

\section{Discussion}

CTPA plays an important role in the diagnosis of PH (18). DECT and SDCT offer novel insights regarding pulmonary perfusion that rival V/Q-Scintigraphy. While it has been shown that IDIs are very valuable for radiologists who have experience in the diagnosis of $\mathrm{PH}$ (15-17), there is only limited experience regarding quantitative analysis of pulmonary perfusion. SDCT offers $3 \mathrm{D}$ volumetric information of iodine distribution in the lungs. We evaluated the usefulness of this information in patients with CTEPH.

We found that automatic lung segmentation and threshold-based segmentation and quantification of 


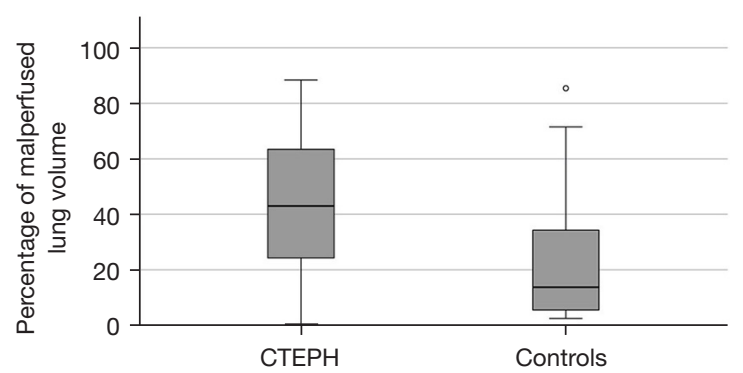

Figure 2 Comparison of the percentage of malperfused lung volume between patients with chronic thromboembolic pulmonary hypertension $(\mathrm{CTEPH})(43.25 \% \pm 24.72 \%)$ and controls $(21.82 \% \pm 20.72 \%)$. Patients with CTEPH had significant $(\mathrm{P}=0.001)$ higher percentage of malperfused lung volume compared to controls.

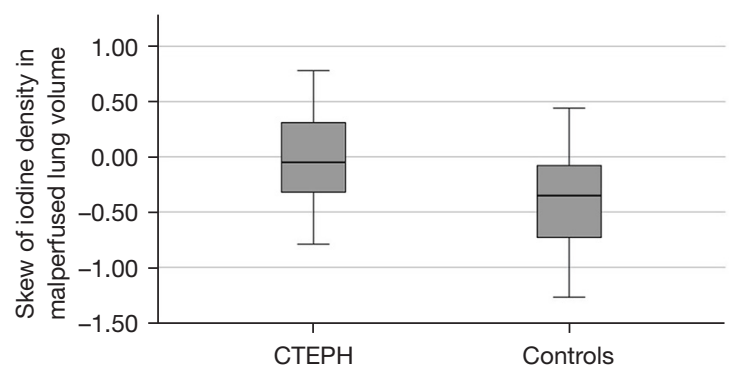

Figure 3 Comparison of skew of iodine density in malperfused lung volume. Controls had a negative skew of iodine density $(-0.382 \pm 0.435)$ while the distribution of iodine density in voxels was more centered in patients with chronic thromboembolic pulmonary hypertension $(\mathrm{CTEPH})(-0.010 \pm 0.396)$. Meaning that in areas of malperfusion controls tended to have voxels with higher iodine density compared to patients with CTEPH. This difference was statistically significant $(\mathrm{P}=0.004)$.

Table 1 Overview of volumetric parameters for patients with chronic thromboembolic pulmonary hypertension and controls

\begin{tabular}{|c|c|c|c|c|c|c|}
\hline & CTEPH & $\mathrm{BCa} 95 \% \mathrm{Cl}$ & Controls & $\mathrm{BCa} 95 \% \mathrm{Cl}$ & $P$ value & Cohen's d \\
\hline Percentage of vessel volume & $2.18 \pm 0.95$ & $1.82-2.53$ & $2.33 \pm 1.29$ & $1.84-2.81$ & 0.882 & -0.13 \\
\hline Mean iodine density in malperfused lung areas $(\mathrm{mg} / \mathrm{mL})$ & $0.023 \pm 0.006$ & $0.021-0.025$ & $0.026 \pm 0.005$ & $0.024-0.028$ & $0.047^{*}$ & -0.54 \\
\hline $\begin{array}{l}\text { Mean iodine density in normal perfused lung areas } \\
(\mathrm{mg} / \mathrm{mL})\end{array}$ & $0.105 \pm 0.033$ & $0.092-0.117$ & $0.117 \pm 0.030$ & $0.106-0.128$ & $0.046^{*}$ & -0.38 \\
\hline Skewness in malperfused lung areas & $-0.010 \pm 0.396$ & $-0.158-0.138$ & $-0.382 \pm 0.435$ & -0.545 to -0.220 & $0.004^{*}$ & -0.89 \\
\hline Mean pulmonary artery pressure (mPAP) (mmHg) & $43.32 \pm 11.30$ & $39.47-47.30$ & n.a. & n.a. & n.a. & n.a. \\
\hline Pulmonary vascular resistance (PVR) (WU) & $8.12 \pm 3.78$ & $6.75-9.70$ & n.a. & n.a. & n.a. & n.a. \\
\hline
\end{tabular}

Data are given as mean \pm standard deviation and $95 \%$ bias-corrected and accelerated confidence interval. *, statistically significant differences.

malperfused lung based on iodine density values is feasible. It reveals significant differences between patients with and without CTEPH. The percentage of malperfused lung volume can be used to predict whether a patient has CTEPH with modest sensitivity and sufficient specificity. While it has been shown in a previous study that differentiation of CTEPH patients is possible on qualitative assessment of IDIs for radiologists with experience in the imaging of $\mathrm{PH}(17)$, these quantitative findings might be helpful to facilitate confident diagnosis of CTEPH for less experienced radiologists. However, the diagnostic performance of the percentage of malperfused lung volume is only mediocre possibly because some pulmonary perfusion changes are lost when only analyzing overall changes in lung perfusion. Thus, more detailed analysis using sophisticated features (Radiomics analysis) which should be possible using this volumetric assessment might be useful.

We found that the extent of pulmonary malperfusion correlates with hemodynamic markers of disease severity. Takagi et al. reported a similar correlation between the mPAP and PVR and the extent of malperfusion scored 


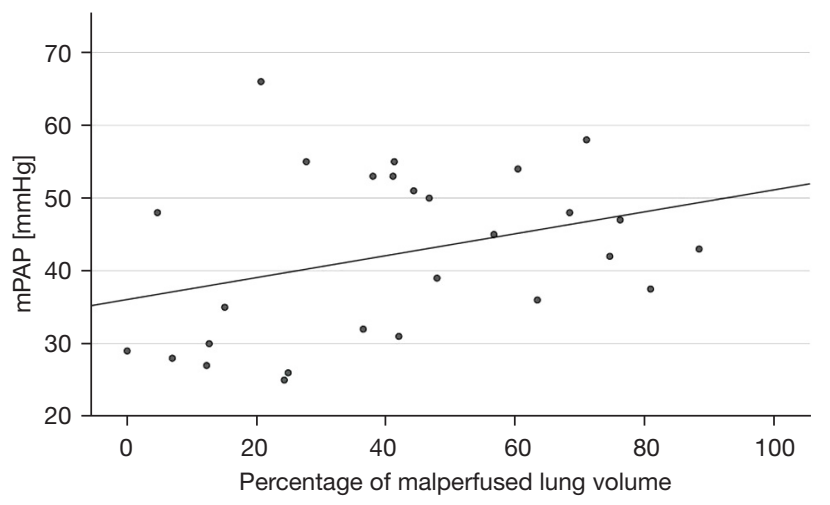

Figure 4 Scatterplot of percentage of malperfused lung volume and mean pulmonary artery pressure (mPAP) for patients with chronic thromboembolic pulmonary hypertension (CTPEH). Percentage of malperfused lung volume tended to be higher in patients with higher mPAP but the correlation was not significant $(\rho=0.34, P=0.064)$.

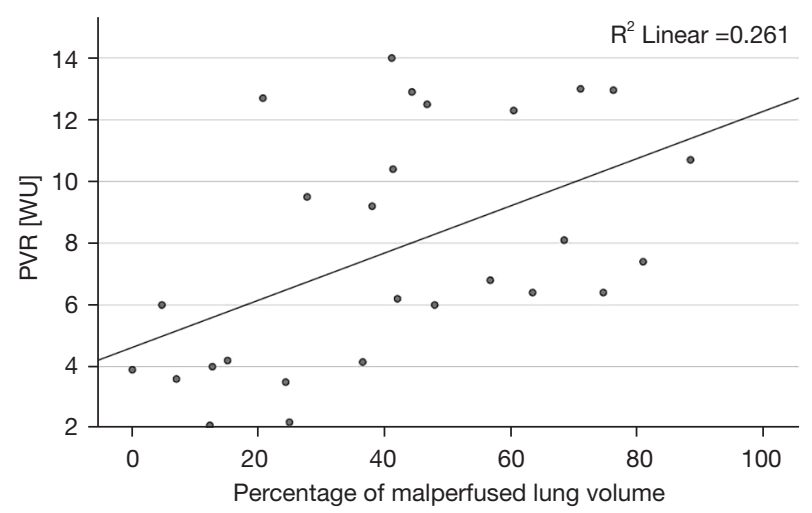

Figure 5 Scatterplot of percentage of malperfused lung volume and pulmonary vascular resistance (PVR) for patients with chronic thromboembolic pulmonary hypertension (CTEPH) Percentage of malperfused lung volume was higher in patients with higher PVR with a significant correlation $(\mathrm{r}=0.57, \mathrm{P}=0.001)$.

by two radiologists in CTEPH using a semi-quantitative approach (19). While we did observe a similar trend in our cohort the correlation between mPAP and the extent of malperfusion did not reach significance in our study, but the correlation between PVR and the extent of malperfusion we found was of modest strength but significant and somewhat higher in our cohort. A potential explanation for these differences may be the significantly shorter delay of only 1 second between measurement of iodine attenuation increase

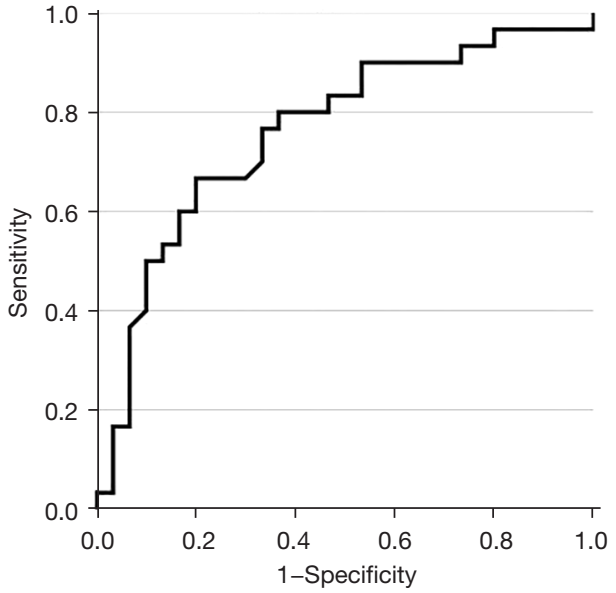

Figure 6 ROC-curve showing the performance for the prediction of chronic thromboembolic pulmonary hypertension by using the percentage of malperfused lung volume (AUC: 0.760).

in the pulmonary artery and the start of the CT scan employed by Takagi et al. Unfortunately, the minimal scan delay possible with the SDCT platform is $4.9 \mathrm{~s}$ which might lead to a decreased sensitivity for malperfused regions on IDIs.

A single study performed an automatic evaluation of pulmonary perfusion by an algorithm called pulmonary perfused blood volume which calculates the relative enhancement of the pulmonary parenchyma compared to the enhancement of the pulmonary artery and provides relative values for the upper, middle and lower lung zone in patients with CTEPH (20). These values correlated significantly with the mPAP (20) but not with the pulmonary resistance. Still these results are similar to our results in showing that the degree of malperfusion in patients with CTEPH seems to be connected to the degree of hemodynamic abnormalities. Our results are in contrast to a previous study which found no significant correlation between mPAP or PVR and the extent of malperfusion scored by visual analysis of two radiologists (17), showing that volumetric quantification provides benefits compared to semiquantitative scoring. Additional benefits of the semiautomated volumetric quantification are its impartiality and time efficiency.

We found that patients with CTEPH showed significantly decreased mean iodine density in areas of malperfused and normal perfused lung as well as in the vessel volume though only by a small margin. Still this 
result may indicate an overall reduced lung perfusion in patients with CTEPH.

While iodine density in malperfused areas was relatively uniformly distributed in patients with CTEPH in controls we saw a significant negative skew, meaning that iodine values tended to be higher. This shows that areas defined as malperfused in controls still had more voxels with a relatively high iodine content. This highlights the difficulty of setting a true cut-off for defining malperfused volumes.

Interestingly, based on our chosen thresholds for differentiating between normal and malperfused lung regions, our control cohort exhibited some degrees of pulmonary malperfusion, too. It is important to note that our control cohort did not consist of healthy individuals but of patients who underwent CTPA for suspected acute pulmonary embolism. Even though PE was excluded, these patients underwent CTPA because of clinical suspicion of $\mathrm{PE}$ and thus might exhibit altered pulmonary perfusion, due to pneumonia, venous congestion, underlying pulmonary emphysema or fibrosis. However, we would deem a CT examination in truly healthy individuals unethical.

The need to define a threshold based on visual assessment is a limitation of our approach. However, it has been shown that the areas of malperfusion on IDIs correlate well with perfusion imaging by V/Q-SPECT $(14,21)$.

Another limitation of our approach are potential inaccuracies of the threshold-based segmentation due to image artifacts. Residual amounts of high-density contrast media in the subclavian veins or the superior vena cava, for example, can lead to falsely increased iodine attenuation values in the upper lung zones. At the same time, artificially reduced densities may occur due to beam hardening effects.

A limitation of the SDCT is that IDIs can only be created by scanning with a tube current of $120 \mathrm{kV}$. Lower tube currents like $100 \mathrm{kV}$ are often used in CTPA to enhance the iodine contrast while simultaneously reducing radiation dose. SDCT allows for reconstruction of monoenergetic images (e.g., at $100 \mathrm{keV}$ ) which improves iodine enhancement if needed.

To conclude, we showed that volumetric assessment of iodine content and thus volumetric quantification of pulmonary malperfusion may assist diagnosis of CTEPH. Our study provides the first results of a truly voxel-based volumetric quantification of iodine content in the lungs. The correlation of hemodynamic parameters and the extent of malperfusion holds promise to provide a noninvasive, objective parameter of disease severity.

\section{Acknowledgments}

Funding: None.

\section{Footnote}

Reporting Checklist: The authors have completed the STROBE reporting checklist. Available at https://dx.doi. org/10.21037/qims-21-229

Conflicts of Interest: All authors have completed the ICMJE uniform disclosure form (available at https://dx.doi. org/10.21037/qims-21-229). GP and SK are employees of Philips. SR reports remunerations for lectures and/ or consultancy from Abbott, Acceleron, Actelion, Arena, Bayer, BMS, Ferrer, Janssen, MSD, Novartis, Pfizer, United Therapeutics, and Vifor; grants to institution from Actelion, AstraZeneca, Bayer, Janssen, and Novartis. RJG received research support from Philips. FG reports payment for expert testimony and support for attending meetings and/or travel by Johnson \& Johnson. JRK reports received research support by Philips and support for attending meetings and/ or travel by Veryan. The other authors have no conflicts of interest to declare.

Ethical Statement: The authors are accountable for all aspects of the work in ensuring that questions related to the accuracy or integrity of any part of the work are appropriately investigated and resolved. The study was conducted in accordance with the Declaration of Helsinki (as revised in 2013). This retrospective study was approved by the Ethics Commission of Cologne University's Faculty of Medicine and the need for informed consent for study enrollment was waived due to the retrospective nature of this study.

Open Access Statement: This is an Open Access article distributed in accordance with the Creative Commons Attribution-NonCommercial-NoDerivs 4.0 International License (CC BY-NC-ND 4.0), which permits the noncommercial replication and distribution of the article with the strict proviso that no changes or edits are made and the original work is properly cited (including links to both the formal publication through the relevant DOI and the license). See: https://creativecommons.org/licenses/by-nc-nd/4.0/.

\section{References}

1. Simonneau G, Gatzoulis MA, Adatia I, Celermajer D, 
Denton C, Ghofrani A, Gomez Sanchez MA, Krishna

Kumar R, Landzberg M, Machado RF, Olschewski H, Robbins IM, Souza R. Updated clinical classification of pulmonary hypertension. J Am Coll Cardiol 2013;62:D34-41.

2. Ruggiero A, Screaton NJ. Imaging of acute and chronic thromboembolic disease: state of the art. Clin Radiol 2017;72:375-88.

3. Ng CS, Wells AU, Padley SP. A CT sign of chronic pulmonary arterial hypertension: the ratio of main pulmonary artery to aortic diameter. J Thorac Imaging 1999;14:270-8.

4. Wilkens H, Konstantinides S, Lang IM, Bunck AC, Gerges M, Gerhardt F, et al. Chronic thromboembolic pulmonary hypertension (CTEPH): Updated Recommendations from the Cologne Consensus Conference 2018. Int J Cardiol 2018;272S:69-78.

5. Galiè N, Humbert M, Vachiery JL, Gibbs S, Lang I, Torbicki A, et al. 2015 ESC/ERS Guidelines for the diagnosis and treatment of pulmonary hypertension: The Joint Task Force for the Diagnosis and Treatment of Pulmonary Hypertension of the European Society of Cardiology (ESC) and the European Respiratory Society (ERS): Endorsed by: Association for European Paediatric and Congenital Cardiology (AEPC), International Society for Heart and Lung Transplantation (ISHLT). Eur Heart J 2016;37:67-119.

6. Simonneau G, Montani D, Celermajer DS, Denton CP, Gatzoulis MA, Krowka M, Williams PG, Souza R. Haemodynamic definitions and updated clinical classification of pulmonary hypertension. Eur Respir J 2019;53:1801913.

7. Devis P, Knuttinen MG. Deep venous thrombosis in pregnancy: incidence, pathogenesis and endovascular management. Cardiovasc Diagn Ther 2017;7:S309-19.

8. Kim NH, Delcroix M, Jenkins DP, Channick R, Dartevelle P, Jansa P, Lang I, Madani MM, Ogino H, Pengo V, Mayer E. Chronic thromboembolic pulmonary hypertension. J Am Coll Cardiol 2013;62:D92-9.

9. Tunariu N, Gibbs SJ, Win Z, Gin-Sing W, Graham A, Gishen P, Al-Nahhas A. Ventilation-perfusion scintigraphy is more sensitive than multidetector CTPA in detecting chronic thromboembolic pulmonary disease as a treatable cause of pulmonary hypertension. J Nucl Med 2007;48:680-4.

10. Ende-Verhaar YM, Meijboom LJ, Kroft LJM, Beenen LFM, Boon GJAM, Middeldorp S, Nossent EJ, Symersky P, Huisman MV, Bogaard HJ, Vonk Noordegraaf A,
Klok FA. Usefulness of standard computed tomography pulmonary angiography performed for acute pulmonary embolism for identification of chronic thromboembolic pulmonary hypertension: results of the InShape III study. J Heart Lung Transplant 2019;38:731-8.

11. Dournes G, Verdier D, Montaudon M, Bullier E, Rivière A, Dromer C, Picard F, Billes MA, Corneloup O, Laurent F, Lederlin M. Dual-energy CT perfusion and angiography in chronic thromboembolic pulmonary hypertension: diagnostic accuracy and concordance with radionuclide scintigraphy. Eur Radiol 2014;24:42-51.

12. Thieme SF, Becker CR, Hacker M, Nikolaou K, Reiser MF, Johnson TR. Dual energy CT for the assessment of lung perfusion--correlation to scintigraphy. Eur J Radiol 2008;68:369-74.

13. Nakazawa T, Watanabe Y, Hori Y, Kiso K, Higashi M, Itoh T, Naito H. Lung perfused blood volume images with dual-energy computed tomography for chronic thromboembolic pulmonary hypertension: correlation to scintigraphy with single-photon emission computed tomography. J Comput Assist Tomogr 2011;35:590-5.

14. Masy M, Giordano J, Petyt G, Hossein-Foucher C, Duhamel A, Kyheng M, De Groote P, Fertin M, Lamblin N, Bervar JF, Remy J, Remy-Jardin M. Dual-energy CT (DECT) lung perfusion in pulmonary hypertension: concordance rate with V/Q scintigraphy in diagnosing chronic thromboembolic pulmonary hypertension (CTEPH). Eur Radiol 2018;28:5100-10.

15. Kim EY, Seo JB, Oh SY, Lee CW, Hwang HJ, Lee SM, Lee YK. Assessment of perfusion pattern and extent of perfusion defect on dual-energy CT angiography: correlations between the causes of pulmonary hypertension and vascular parameters. Korean J Radiol 2014;15:286-94.

16. Giordano J, Khung S, Duhamel A, Hossein-Foucher C, Bellèvre D, Lamblin N, Remy J, Remy-Jardin M. Lung perfusion characteristics in pulmonary arterial hypertension $(\mathrm{PAH})$ and peripheral forms of chronic thromboembolic pulmonary hypertension (pCTEPH): Dual-energy CT experience in 31 patients. Eur Radiol 2017;27:1631-9.

17. Kröger JR, Gerhardt F, Dumitrescu D, Rosenkranz S, Schmidt M, Maintz D, Bunck AC. Diagnosis of pulmonary hypertension using spectral-detector CT. Int J Cardiol 2019;285:80-5.

18. Dong C, Zhou M, Liu D, Long X, Guo T, Kong X. Diagnostic accuracy of computed tomography for chronic thromboembolic pulmonary hypertension: a systematic review and meta-analysis. PLoS One 2015;10:e0126985. 
19. Takagi H, Ota H, Sugimura K, Otani K, Tominaga J, Aoki T, Tatebe S, Miura M, Yamamoto S, Sato H, Yaoita N, Suzuki H, Shimokawa H, Takase K. Dual-energy CT to estimate clinical severity of chronic thromboembolic pulmonary hypertension: Comparison with invasive right heart catheterization. Eur J Radiol 2016;85:1574-80.

20. Meinel FG, Graef A, Thierfelder KM, Armbruster M, Schild C, Neurohr C, Reiser MF, Johnson TR. Automated quantification of pulmonary perfused blood volume by

Cite this article as: Kroeger JR, Zöllner J, Gerhardt F, Rosenkranz S, Gertz RJ, Kerszenblat S, Pahn G, Maintz D, Bunck AC. Detection of patients with chronic thromboembolic pulmonary hypertension by volumetric iodine quantification in the lung - a case control study. Quant Imaging Med Surg 2022;12(2):1121-1129. doi: 10.21037/qims-21-229 dual-energy CTPA in chronic thromboembolic pulmonary hypertension. Rofo 2014;186:151-6.

21. Tang CX, Yang GF, Schoepf UJ, Han ZH, Qi L, Zhao YE, Wu J, Zhou CS, Zhu H, Stubenrauch AC, Mangold S, Zhang LJ, Lu GM. Chronic thromboembolic pulmonary hypertension: Comparison of dual-energy computed tomography and single photon emission computed tomography in canines. Eur J Radiol 2016;85:498-506. 